\title{
IMPURITY-SCATTERING LIMITED ELECTRON MOBILITY IN FREE STANDING QUANTUM WIRES: IMAGE CHARGE EFFECT
}

\author{
P. VAGNER AND M. MošKo \\ Institute of Electrical Engineering, Slovak Academy of Sciences \\ Dúbravská cesta 9, 84239 Bratislava, Slovakia
}

\begin{abstract}
We calculate the impurity-scattering limited mobility of the one-dimensional electron gas in a rectangular GaAs quantum wire confined in the vertical (growth) direction by $n$-modulation doped AlGaAs layers and free standing along the transverse direction. The scattering potential of the ionized impurity is obtained by solving the Poisson equation with $z$-dependent electrostatic permittivity in order to take into account the image charge effect due to the abrupt permittivity change at the GaAs/air interfaces. We show that the "image impurity" scattering tends to drastically reduce the electron mobility for sufficiently small $(\approx 10 \mathrm{~nm})$ transverse wire widths.
\end{abstract}

PACS numbers: 73.50.-h, 73.50.Bk

The nanolithographic patterning and etching of layered AlGaAs/GaAs/ AlGaAs structures have made possible to fabricate a rectangular GaAs quantum wire confined in the vertical (growth) direction ( $y$ ) by AlGaAs layers, and free standing along the transverse direction (z) [1]. Nowadays there is a trend to produce free standing wires with transverse widths close to $10 \mathrm{~nm}$ in order to enhance the quantum confinement of the carriers. In this paper we calculate the impurity-scattering limited electron mobility in free standing GaAs wires. The scattering potential of the ionized impurity is obtained by solving the Poisson equation with $z$-dependent electrostatic permittivity in order to take into account the image charge effect due to the abrupt permittivity change at the GaAs/air interfaces. We compare the electron mobility in the free standing GaAs wire with the mobility in the GaAs wire buried in the AlGaAs material, in which the image charge is negligible. Due to scattering by the image potentials of ionized impurities, the electron mobility in free standing quantum wires of small enough $(\approx 10 \mathrm{~nm})$ transverse width is found to be intrinsically limited to a value substantially lower than in buried quantum wires.

We consider an undoped GaAs wire of the infinite length in the $x$ direction and of the rectangular cross-section in the $y-z$ plane. The wire is confined in the vertical $(y)$ direction by AlGaAs layers. In the transverse $(z)$ direction 
the whole layered structure is confined by vacuum. The GaAs/AlGaAs interfaces are positioned at $y=0$ and $y=L_{y}$ ( $L_{y}$ is the vertical wire width), the $\mathrm{GaAs}(\mathrm{AlGaAs}) /$ vacuum interfaces are positioned at $z=0$ and $z=L_{z}\left(L_{z}\right.$ is the transverse wire width). In each of the confining AlGaAs layers a sublayer of the vertical width $L_{d}$ is doped to the donor density $N_{\text {imp. }}$. To increase the electron mobility, the sublayer is separated from the GaAs wire by an undoped AlGaAs spacer layer of the vertical width $L_{\text {sp. }}$. Assuming a periodic GaAs/AlGaAs structure with all donors ionized, the linear electron density in the GaAs wire should be $n_{L}=N_{\mathrm{imp}} L_{z} L_{d}$. In reality, most of the electrons is depleted by interface states at the GaAs(AlGaAs)/vacuum interfaces [1], i.e., $n_{L} \ll N_{\text {imp }} L_{z} L_{d}$. We consider in our calculations $n_{L}=\frac{1}{10}\left(N_{\mathrm{imp}} L_{z} L_{d}\right)$, which is more relevant to the situation when electrons are excited from the interface states by light (or to the case of semiconductors like InGaAs, where the depletion due to interfaces is much weaker). Since there is only one free electron per ten ionized donors, we neglect the free carrier screening of donor potentials.

At the $\mathrm{GaAs}(\mathrm{AlGaAs}) /$ vacuum interfaces (i.e, at $z=0$ and $z=L_{z}$ ) there is an abrupt permittivity change from $\varepsilon_{s}=13 \varepsilon_{0}$ to $\varepsilon_{0}$, where $\varepsilon_{s}$ and $\varepsilon_{0}$ is the permittivity of $\mathrm{GaAs}(\mathrm{AlGaAs})$ and vacuum, respectively (for simplicity, in the $\mathrm{AlGaAs}$ we take the same $\varepsilon_{s}$ as in the GaAs). To calculate the scattering potential $V$ of the ionized impurity inside the quantum wire, we solve the Poisson equation

$$
\begin{aligned}
& \nabla\left[\varepsilon(z) \nabla V\left(r, r_{0}\right)\right]=-q_{0} \delta\left(r-r_{0}\right), \\
& \varepsilon(z)=\varepsilon_{0}+\left(\varepsilon_{s}-\varepsilon_{0}\right) \theta(z) \theta\left(L_{z}-z\right),
\end{aligned}
$$

where $r_{0}$ and $q_{0}$ are the impurity position and the impurity charge, respectively, $\theta$ is the heavyside step function, and $\varepsilon(z)$ is the position-dependent permittivity. The Fourier-transformed potential, $V\left(Q, z, z_{0}\right)=\int \mathrm{d}\left(x-x_{0}\right) \int \mathrm{d}\left(y-y_{0}\right)$ $\times \exp \left\{\mathrm{i}\left[Q_{x}\left(x-x_{0}\right)+Q_{y}\left(y-y_{0}\right)\right]\right\} V\left(r, r_{0}\right)$, can be obtained from (1) analytically in the form [2]

$$
\begin{aligned}
& V\left(Q, z, z_{0}\right)=\frac{q_{0}}{2 \varepsilon_{s} Q} \exp \left(-Q\left|z-z_{0}\right|\right) \\
& +\frac{q_{0}}{2 \varepsilon_{s} Q} \frac{\varepsilon_{s}-\varepsilon_{0}}{2 \varepsilon_{0}}\left[\chi_{1}\left(Q, z, z_{0}\right)+\chi_{2}\left(Q, z, z_{0}\right)\right] \\
& \chi_{1}\left(Q, z, z_{0}\right)=\exp (-Q z) \frac{\exp \left(-Q z_{0}\right)+\frac{\varepsilon_{s}-\varepsilon_{0}}{\varepsilon_{s}+\varepsilon_{0}} \exp \left(-Q\left(2 L_{z}-z_{0}\right)\right)}{1+\frac{\varepsilon_{s}-\varepsilon_{0}}{2 \varepsilon_{0}}\left(1-\frac{\varepsilon_{s}-\varepsilon_{0}}{\varepsilon_{s}+\varepsilon_{0}} \exp \left(-2 Q L_{z}\right)\right)} \\
& \left.\chi_{2}\left(Q, z, z_{0}\right)=\mathrm{e}^{-Q\left(L_{z}-z\right)} \frac{\exp \left(-Q\left(L_{z}-z_{0}\right)\right)+\frac{\varepsilon_{s}-\varepsilon_{0}}{\varepsilon_{s}+\varepsilon_{0}} \exp \left(-Q\left(L_{z}+z_{0}\right)\right)}{1+\frac{\varepsilon_{s}-\varepsilon_{0}}{2 \varepsilon_{0}}\left(1-\frac{\varepsilon_{s}-\varepsilon_{0}}{\varepsilon_{s}+\varepsilon_{0}} \exp \left(-2 Q L_{z}\right)\right)}\right)
\end{aligned}
$$

where $Q=\sqrt{Q_{x}^{2}+Q_{y}^{2}}$. The first term on the right-hand side of (2) is the Coulomb potential of the impurity and the second term is its image potential.

In the calculations only the lowest energy subband is assumed to be occupied by electrons, since we deal with low lattice temperatures $(4.2 \mathrm{~K}$ and $77 \mathrm{~K})$ and low electron densities $\left(\approx 1 \times 10^{5}-5 \times 10^{7} \mathrm{~m}^{-1}\right)$. The electron envelope function is 
taken as $\phi(y, z)=\frac{2}{\sqrt{L_{y} L_{z}}} \sin \left(\pi y / L_{y}\right) \sin \left(\pi z / L_{z}\right)$. We do not take into account the band bending created by remote donors and interface states and we also ignore the renormalization of the envelope function due to electron image charge. The electron mobility, $\mu$, can be obtained from the momentum relaxation time formulation of the Boltzmann transport equation

$$
\mu=-\frac{e \hbar^{2}}{n_{L} m^{2}} \int_{-\infty}^{\infty} \frac{\mathrm{d} k}{\pi} k^{2} \tau(k) \frac{\partial f_{0}(\varepsilon(k))}{\partial \varepsilon},
$$

where $\varepsilon=\hbar^{2} k^{2} /(2 m)$ is the electron energy, $k$ is the wave vector in the $x$-direction, and $m=0.067 m_{0}$ is the effective mass. The momentum relaxation time $\tau(k)$ can be derived from the Fermi golden rule as

$$
\begin{aligned}
& \frac{1}{\tau(k)}=\frac{2 m}{\hbar^{3}} \int_{-\infty}^{\infty} \mathrm{d} y_{0} \int_{0}^{L_{x}} \mathrm{~d} z_{0} N\left(y_{0}\right) \frac{\left|H\left(Q_{x}=2 k\right)\right|^{2}}{|k|}, \\
& H\left(Q_{x}=2 k\right)=e \int_{0}^{L_{y}} \mathrm{~d} y \int_{0}^{L_{x}} \mathrm{~d} z|\phi(y, z)|^{2} \int_{-\infty}^{\infty} \frac{\mathrm{d} Q_{y}}{2 \pi} \\
& \quad \times \exp \left[-i Q_{y}\left(y-y_{0}\right)\right] V\left(Q, z, z_{0}\right),
\end{aligned}
$$

where $e$ is the electron charge and $N\left(y_{0}\right)$ is the ionized impurity density in the structure. $N\left(y_{0}\right)$ involves the above discussed density $\left(N_{\text {imp }}\right)$ of remote donors in the AlGaAs layers and the spatially homogeneous density of background impurities, $N_{\mathrm{bg}}$. In numerical calculations $N_{\mathrm{bg}}=10^{20} \mathrm{~m}^{-3}, L_{d}=10 \mathrm{~nm}$, and $L_{y}=10 \mathrm{~nm}$.

Figure 1 shows the electron mobility versus $L_{z}$ at temperature $4.2 \mathrm{~K}$. The result with (without) image charge is the mobility in the free standing (buried) $\mathrm{GaAs}$ wire. One sees that the effect of image charge is not important for large $L_{z}(50-60 \mathrm{~nm})$. With decreasing $L_{z}$ more impurities become close enough to the $\mathrm{GaAs} /$ vacuum interfaces to create strong image potential (the second term on the right-hand side of $\mathrm{Eq}$. (2)). This causes one order of magnitude decrease in the mobility at $L_{z} \approx 10 \mathrm{~nm}$. Thus, for the technologically achievable transverse wire widths $(10-20 \mathrm{~nm})$ the electron scattering by image impurity potentials is more important than the electron scattering by unperturbed Coulomb impurity potentials. The $L_{z}$ values smaller than $10 \mathrm{~nm}$ are hardly achievable by present technologies [1]. Therefore, the results for $L_{z} \rightarrow 0$ should be viewed as a demonstration of mathematical trends. The mobility without image charge effect tends to diverge for $L_{z} \rightarrow 0$, because for $L_{z} \rightarrow 0$ there are no impurities in the "wire". The mobility with the image charge effect would show the same tendency for smaller $L_{z}$ than those considered in Fig. 1 . The mobility dependence on $N_{\mathrm{imp}}$ and $L_{\mathrm{sp}}$ shows the well-known trends, previously discussed for buried GaAs wires [3-5]. Figure 2 shows the same calculation as Fig. 1, but for a lattice temperature of $77 \mathrm{~K}$. The obtained mobilities are qualitatively similar to those in Fig. 1, but several orders of magnitude higher. We expect that at temperatures $\geq 77 \mathrm{~K}$ the mobility will be determined mainly by electron-phonon interactions and the impurity scattering (including the image potential scattering) will be unimportant. Finally, Fig. 3 compares the contributions from remote donors and background impurities for two various spacer widths, $L_{\mathrm{sp}}$. For $L_{\mathrm{sp}}=5 \mathrm{~nm}$ one can see that the 

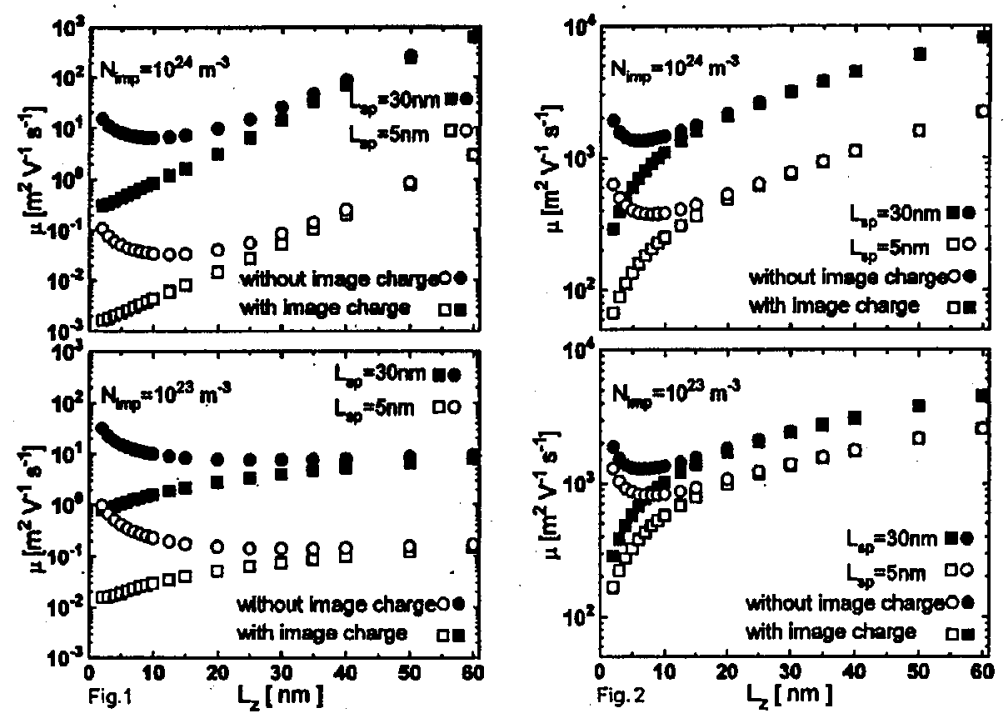

Fig. 1. Electron mobility versus transverse wire width $L_{z}$ at temperature $4.2 \mathrm{~K}$ for two various space layer widths, $L_{\mathrm{sp}}$, and for two various densities of remote donors, $N_{\mathrm{imp}}$. Fig. 2. The same material as in Fig. 1, but at a lattice temperature of $77 \mathrm{~K}$.

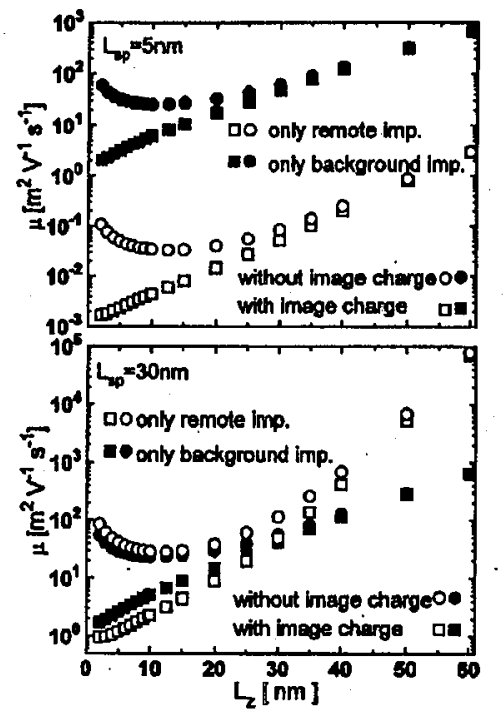

Fig. 3. Electron mobility versus $L_{z}$ at temperature $4.2 \mathrm{~K}$ for $N_{\mathrm{imp}}=10^{24} \mathrm{~m}^{-3}$. The upper (lower) figure shows the results for $L_{\mathrm{sp}}=5 \mathrm{~nm}(30 \mathrm{~nm})$. The calculation restricted to the remote impurity scattering is compared with the calculation restricted to the background impurity scattering. The results with and without image charge are distinguished. 
remote donor scattering dominates, while for $L_{\mathrm{sp}}=30 \mathrm{~nm}$ the background impurity scattering becomes also important. The latter mechanism is overestimated in our calculations, because we neglect the free carrier screening. This is not justified for background impurities, since $N_{\text {bg }}$ is much lower (rather than much higher) than the effective volume density of free carriers.

In conclusion, we have calculated the impurity-scattering limited electron mobility in the free-standing GaAs quantum wire. The scattering by image impurity charges has been considered and shown to strongly reduce the mobility for small transverse wire widths.

This work was supported by the Slovak Grant Agency for Science.

\section{References}

[1] R. Cingolani, R. Rinaldi, Rivista Del Nuovo Cimento 16, 1 (1993).

[2] M. Moško, to be published.

[3] L. Baudry, J.L. Thobel, M. Charef, F. Dessenne, P. Bourel, J. Appl. Phys. 74, 6281 (1993).

[4] G. Fishman, Phys. Rev. B 34, 2394 (1986).

[5] J. Lee, H.N. Spector, J. Appl. Phys 54, 3921 (1983). 